\title{
Evaluación de un puente de vías férrea mediante ensayos estructurales y modelación computacional
}

\author{
Evaluation of a railway bridge through structural tests and computational modelling
}

Fecha de entrega: 16 de junio 2015

Fecha de aceptación: 11 de noviembre 2015

\section{Rafael Ramírez Díaz ${ }^{1}$, Yudiel Gámez Breto ${ }^{1}$, Hamlet Pérez Lecusay ${ }^{1}$ y Ernesto Chagoyén Méndez ${ }^{2}$}

\footnotetext{
${ }^{1}$ Empresa Nacional de Investigaciones Aplicadas, Oficina de Consultoría y Diseño, Calle 100 No. 9107 Entre Vento y 16, Boyeros, La Habana,Cuba,rafael@enia.co.cu,ygamez@enia.co.cu,hperez@enia.co.cu

${ }^{2}$ Universidad Central de las Villas, Facultad de Construcciones, Carretera a Camajuaní kilómetro 51⁄2, Santa Clara, Villa Clara, Cuba, chagoyen@uclv.edu.cu
}

La reanimación de la vía férrea que enlaza la Zona de Desarrollo Integral del Mariel con la Habana es una actividad de crucial importancia para la economía cubana. Entre los elementos que conforman dicha vía se encuentran una serie de puentes, que precisan ser revisados y comprobados estructuralmente, para determinar su capacidad resistente ante las nuevas cargas que sobre ellos actuarán. La investigación se centra en el estudio de la capacidad estructural del puente que atraviesa la Autopista Habana - Pinar del Río. Para ello se aplicó un procedimiento de investigación que comprende la evaluación visual de la estructura, la realización de ensayos de diagnóstico estructural: auscultación ultrasónica, extracción de muestras de hormigón y detección de aceros de refuerzo. Además se realizaron pruebas de carga estáticas y dinámicas y la modelación computacional de la estructura basada en el Método de Elementos Finitos. Se logró determinar el estado actual de los materiales componentes del puente, se identificaron los modos propios de oscilación del puente, el periodo fundamental de vibración $T=0.32 \mathrm{~s}$. A partir de los resultados obtenidos en el proceso de caracterización estructural y con la realización de ensayos de carga se logró la creación de un modelo computacional con un nivel de correspondencia del $96.2 \%$ con la respuesta del elemento estudiado, a partir del cual se identificaron y establecieron los límites de velocidad y carga de los trenes que pueden circular por el puente.

Palabras clave: análisis estructural, pruebas de carga, modelación $M E F$, resonancia lateral del puente
The reactivation of the railroad that connects the Area of Integral Development of Mariel with Havana city is an activity of crucial importance for the Cuban economy. Among the elements that define this railroad, there are a series of bridges, which needs to be structurally checked, aiming to determine their resistance under the action of new loads coming from the exploitation of the Commercial Development Area of Mariel. This work is focused on the study of the structural capacity of the bridge that crosses the Havana-Pinar del Río Motorway. Research procedure includes the recognition and visual evaluation of the elements, tests of structural diagnosis, static and dynamic load tests, and computational modelling of the structure and its ulterior calibration based on the tests developed. As results, the current state of the component materials of the bridge was determined; based on static and dynamic load tests and FEM modelling mode shapes, frequencies and a period of fundamental mode of vibration of $T=0.32 \mathrm{~s}$ were obtained. Also computational calibrated model with a 96.2\% of coincidence with the real behaviour of the structure was developed. Based on such a model, speed exploitation limits and load limits were established for train circulating on the monitored bridge.

Keywords: structural analysis, load testing, FEM modelling, swing bridge resonance

\section{Introducción}

La verificación estructural es un proceso tanto o más complejo que el proceso de diseño estructural, puesto que se debe pronosticar el comportamiento de las estructuras a partir de información que por lo general no se encuentra disponible. Por esta razón y en forma previa a la 
verificación se debe efectuar un minucioso estudio de las propiedades mecánicas de los materiales presentes en la edificación, una prospección de su estado, la verificación de los detalles del refuerzo efectivamente dispuesto en la obra y la corroboración de dimensiones, modificaciones y cargas existentes en la estructura. Durante este proceso de verificación estructural se recurre a diferentes herramientas como la realización de ensayos de caracterización de materiales, la aplicación de ensayos estructurales de tipo estático y dinámico y la modelación computacional por el método de los elementos finitos. Utilizando para la obtención de los resultados varios métodos del análisis estructural entre los que se destacan el análisis estático y dinámico de estructuras.

El análisis estático de estructuras es el proceso sistemático que concluye con el conocimiento de las características de su comportamiento bajo un cierto estado de cargas, las cuales no dependen del tiempo. Se incluye, habitualmente, bajo la denominación genérica de estudio del comportamiento tanto el análisis de los estados tensional y deformacional alcanzados por los elementos y componentes físicos de la estructura como la obtención de conclusiones sobre la influencia recíproca con el ambiente o sobre sus condiciones de seguridad. El análisis dinámico de estructuras se refiere al análisis de las oscilaciones o vibraciones que puede sufrir una estructura alrededor de su posición de equilibrio. El análisis dinámico es importante porque ese movimiento oscilatorio produce una modificación de las tensiones y deformaciones en el tiempo, incluyendo la posibilidad de ocurrencia de resonancia (Kiseliov, 1983).

Otraherramienta fundamental de los estudios de verificación estructural son los ensayos de diagnóstico estructural. Ellos brindan gran parte de la información necesaria para caracterizar una edificación, específicamente desde el punto de vista de la caracterización de los materiales y de la geometría. Entre los ensayos de diagnóstico más utilizados a nivel mundial se encuentran: levantamientos patológicos para detectar, clasificar y cuantificar los deterioros, esclerometría, ultrasonido, extracción de testigos, carbonatación, detección y ubicación de acero de refuerzo, concentración de iones cloruros, concentración de sulfatos, nivel de $\mathrm{pH}$ y pruebas de carga.

Las pruebas de carga son ensayos que se realizan cuando existen incertidumbres sobre la estabilidad, funcionalidad o durabilidad de una estructura, además cuando ha estado sometida a un evento extremo y se tienen dudas acerca de su funcionamiento. Su fin principal es verificar el comportamiento de una estructura ya construida tras la aplicación de una determinada carga, generalmente superior a la de servicio. Como en la práctica no es posible someter a una estructura completa a estos ensayos (a excepción de puentes) la prueba se limita a las zonas más representativas o a las que presentan una patología determinada. Las pruebas de carga estática se aplican y retiran de forma progresiva. Son las ejecutadas en mayor número y frecuentemente son las que se realizan en edificaciones. Su fundamento se basa en la aplicación uniforme y controlada de cargas verticales sobre una determinada superficie, y se obtiene información a través del control de las deformaciones y/o posibles fisuras o defectos que puedan aparecer. Esta prueba es aplicable fundamentalmente a elementos o estructuras que pueden sufrir deformaciones apreciables bajo una determinada carga y cuya rotura se produce tras deformaciones significativas; es decir fluyen antes de un posible colapso.

Las pruebas de carga dinámicas son indicadas para estructuras en las que se prevea un considerable efecto de vibraciones, como ocurre en puentes y en estructuras industriales. Consisten en simular acciones mediante golpes, impactos, el paso de vehículos o personas que logren producir cambios en la estructura para realizar el análisis; posteriormente se analiza la respuesta de dicha estructura mediante la instrumentalización previamente instalada (Ewins, 2000).

El estudio y detección de daños estructurales mediante la utilización de mediciones dinámicas y la complementación utilizando la modelación computacional a través del método de los elementos finitos, es el objetivo de numerosas investigaciones en la actualidad, debido a las aplicaciones prácticas obtenidas (Alvandi y Cremona, 2002). En el caso de Cuba la aplicación de este tipo de estudios es reciente, por lo cual su utilización en los procesos de evaluación estructural resulta novedosa, permitiendo la obtención de resultados más profundos y eficaces, de una manera más rápida y menos invasiva con respecto a la estructura analizada. 


\section{Descripción del puente}

Para el desarrollo de la investigación se tomará como caso de estudio el Puente Ferroviario que atraviesa la Autopista Habana-Pinar (Figura 1). Este puente está ubicado en el vial ferroviario perteneciente a la Zona de Desarrollo Integral del Mariel ZDIM, cuya función es dar continuidad a la vía férrea en la intersección a dos niveles con la Autopista Pinar del Río - Habana. El puente está conformado por cuatro tramos cuyas luces son de $18.20 \mathrm{~m}$ en los tramos sobre la autopista y $12.05 \mathrm{~m}$ en los tramos de los extremos del puente y el mismo posee una altura con respecto a la vía de aproximadamente $4.20 \mathrm{~m}$.

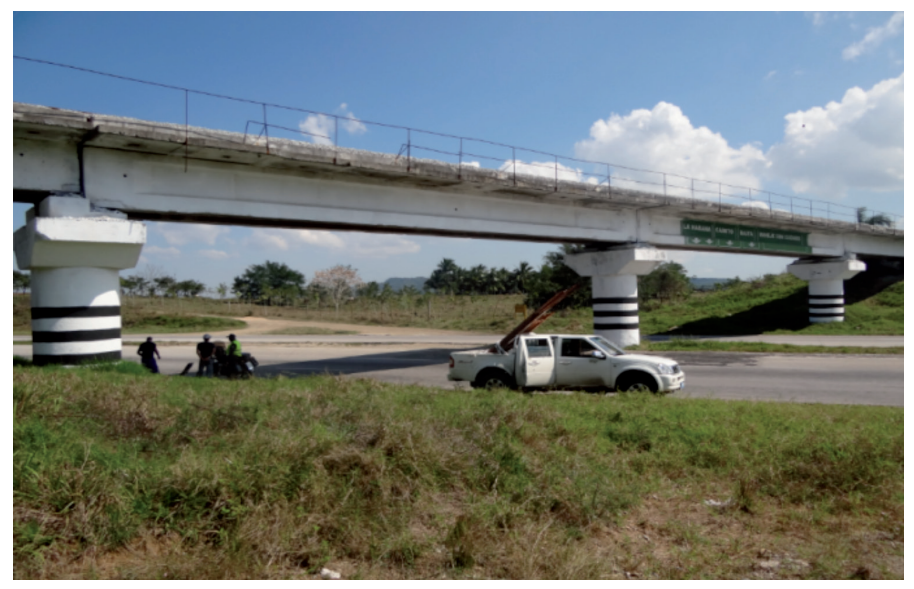

Figura 1: Puente de la Autopista Habana-Pinar

La estructura del puente está conformada por una losa de hormigón armado, la cual sirve de sostén a la vía férrea. Esta losa está conformada por tramos o segmentos de losa discontinuos entre sí, los cuales adquieren cierto grado de continuidad estructural debido a la acción de la capa de balasto que soporta de conjunto con la vía férrea. La losa descansa, sobre vigas pretensadas en las dos luces centrales y sobre vigas armadas en las dos luces exteriores, las cuales transmiten las cargas al suelo por medio de las pilas y los estribos, estando concebidas las uniones entre las vigas y las pilas como articulaciones (ver Figura 2).

En los elementos de hormigón que componen el puente se identificaron a partir de la extracción de testigos diferentes valores de resistencia a compresión, los mismos fueron: $24 \mathrm{MPa}$ en las vigas de hormigón armado, $37 \mathrm{MPa}$ en vigas postesadas y $27 \mathrm{MPa}$ en los cabezales y columnas. Los espesores de recubrimiento de hormigón al acero más expuesto varían entre $2.50 \mathrm{~cm}$ a $7.50 \mathrm{~cm}$. La masa de hormigón no se encuentra afectada por el desarrollo de carbonatación. Los valores de porosidad son inferiores al 10\% que según los criterios de la Red-Durar (1997) indican un hormigón de buena calidad y compacidad. Las concentraciones de iones cloruros son notablemente inferiores a $0.05 \%$ que es el valor límite establecido. Estas características permiten identificar una masa de hormigón sana y durable.

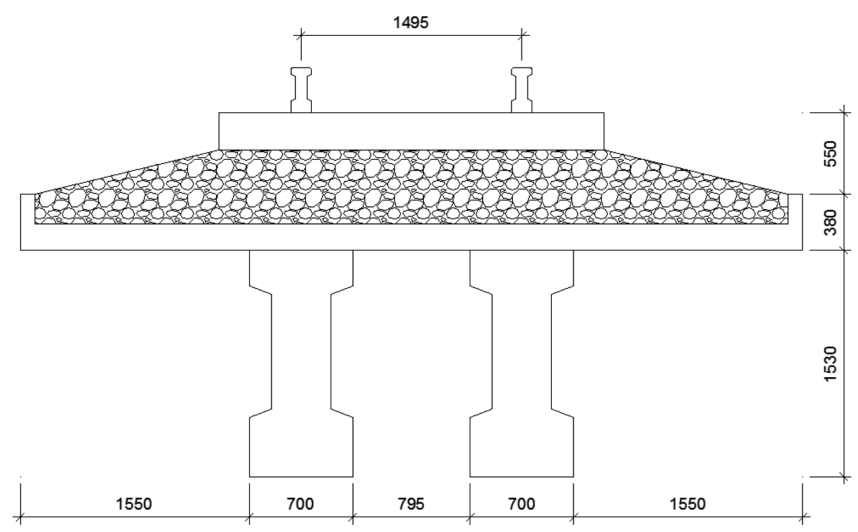

Figura 2: Esquema de la sección transversal del puente (dimensiones en $\mathrm{mm}$ )

A partir de la caracterización de los elementos de refuerzo del puente se logró determinar que las vigas pretensadas están reforzadas por medio de 5 torones de 12 cables de $7 \mathrm{~mm}$ de diámetro cuya excentricidad es de $66 \mathrm{~cm}$. Estos transfieren una fuerza de pretensado inicial $P_{0}=3777 \mathrm{kN}$ y una fuerza de pretensado final $P_{\mathrm{f}}=3147 \mathrm{kN}$, lo cual dota a la sección del elemento de una capacidad nominal de resistencia a la flexión, $M_{\mathrm{n}}=3800 \mathrm{kNm}$. Además en estas vigas existe un refuerzo adicional de 4 barras de acero de $25 \mathrm{~mm}$ de diámetro, lo que le confiere a la pieza una capacidad adicional de resistencia a la flexión $M_{\mathrm{n}}=700$ $\mathrm{kNm}$, confiriéndole a la viga una capacidad resistente total a la flexión de $M_{\mathrm{n}}=4500 \mathrm{kNm}$. Las vigas de hormigón armado se encuentran reforzadas con 20 barras de acero de $25 \mathrm{~mm}$ de diámetro, que le confieren una capacidad resistente a flexión, $M_{\mathrm{n}}=3600 \mathrm{kNm}$.

\section{Protocolo de investigación}

El desarrollo de la investigación comprende a partir del conocimiento de las características fundamentales de la estructura (materiales componentes, geometría, cargas y condiciones de apoyo) la aplicación de ensayos de carga que permitan caracterizar el comportamiento del puente 
tanto bajo cargas estáticas como dinámicas para lo cual se definieron dos familias de pruebas a realizar. La primera familia corresponde a pruebas de carga estática que permiten identificar las relaciones carga-deformación que caracterizan la respuesta de la estructura ante la acción de las cargas. La segunda familia corresponde a las pruebas de carga dinámicas, este tipo de pruebas permite identificar las principales características vibratorias de la estructura.

Para complementar los resultados de la investigación fue creado un modelo computacional en $3 \mathrm{D}$, basado en la utilización del Método de Elementos Finitos MEF, mediante el software Midas Civil (2013), tomando como base los planos originales de construcción, así como los resultados de los estudios de diagnóstico realizados. Este modelo fue validado a partir de los resultados de las pruebas realizadas, y posteriormente a su validación o ajuste a la realidad de respuesta de la estructura fueron evaluadas diferentes hipótesis de esfuerzos a los que puede ser sometido el puente.

El MEF es un método de solución de problemas ingenieriles por aproximación, en donde la región de integración que generalmente es continua, se discretiza, de forma que el elemento continuo se divide en un número finito de partes, cuyo comportamiento se especifica mediante un número finito de parámetros asociados a ciertos puntos característicos denominados nodos. Estos nodos son los puntos de unión de cada elemento con sus adyacentes. La solución del sistema completo, en el que se involucran las ecuaciones de equilibrio, de compatibilidad de desplazamientos, las restricciones de los apoyos y constricciones, sigue entonces las reglas de los problemas discretos. El sistema completo se forma por ensamblaje de los elementos. Las incógnitas en el interior de cada elemento quedan definidas a partir del comportamiento de los nodos mediante las adecuadas funciones de interpolación o funciones de forma.

Este método, por tanto, se basa en transformar un cuerpo de naturaleza continua en un modelo discreto aproximado, esta transformación se denomina discretización. El conocimiento de lo que sucede en el interior de este modelo de cuerpo aproximado, se obtiene mediante la interpolación de los valores conocidos en los nodos. Es por tanto una aproximación de los valores de una función a partir del conocimiento de un número determinado y finito de puntos.
Como parte de la evaluación de las características vibratorias del puente se recurrió a métodos de identificación modal para determinar y valorar las frecuencias propias de oscilación y sus correspondientes formas modales. La identificación modal fue llevada a cabo mediante la aplicación del método de Lanczos (1950). El método Lanczos es un método de interacción numérico, el cual mediante el análisis por medio de elementos finitos se utiliza para la identificación de frecuencias y modos propios de vibración en problemas de dinámica estructural. Este método es muy eficiente en la solución de problemas que implican a estructuras con alto grado de dificultad (estructuras con alto grado de hiperestaticidad, con elementos de diferentes materiales y geometría irregular). En la actualidad el método ha demostrado su fuerza con respecto a otros métodos utilizados con el mismo fin y ha sido implementado como base de cálculo para la determinación de los modos propios de oscilación de edificaciones en numerosos softwares comerciales destinados al análisis y diseño de estructuras (Bathe, 2012). Entre las diversas ventajas que presenta este método de análisis modal, se destaca que permite la obtención de un número arbitrario de soluciones en el problema de identificación modal, mediante la utilización de una sola configuración de las iteraciones, sin reducir el orden de la matriz de análisis (Lanczos, 1950).

La aplicación de las cargas fue mediante la acción de una locomotora de fabricación china, modelo DF7g-C de 123 ton de masa. Esta locomotora es utilizada para la tracción de trenes de carga y de pasajeros, la misma posee una potencia de carga, brindada por su motor diésel de 1840 $\mathrm{kW}$ mediante transmisión eléctrica, lo cual le permite desarrollar una velocidad máxima de $120 \mathrm{~km} / \mathrm{h}$. Esta máquina posee una galga (ancho de vía) de $1495 \mathrm{~mm}$ y tributa a la estructura portante de la vía $205 \mathrm{kN}$ por eje de carga a través de 6 ejes de carga (Figura 3 ).

Para el monitoreo de la estructura fue utilizado el Radar Interferométrico IBIS-FS, el cual es un equipo que permite el monitoreo dinámico y estático de estructuras. Este equipo permite el monitoreo a distancia de estructuras con una precisión de centésima de milímetro en las mediciones $\mathrm{y}$ un rápido, fácil y completo proceso de medición de parámetros estáticos y dinámicos con una frecuencia de muestreo en un rango de 100 a $200 \mathrm{~Hz}$, de diferentes tipologías de obras constructivas (IDS-Ingenieria del 


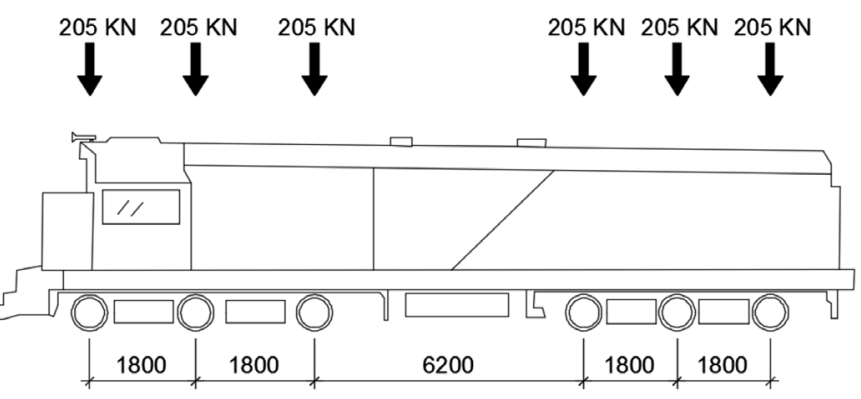

Figura 3: Esquema de la locomotora DF7g-C (dimensiones en $\mathrm{mm}$ )

Sistemi, 2013). La interferometría es una técnica de medición por medio de radar la cual consiste en la medición de desplazamientos de objetos mediante la comparación de fases de información de las ondas electromagnéticas reflejadas por el objeto en diferentes momentos de tiempo. Generalmente las ondas electromagnéticas reflejadas por un objeto difieren al menos en términos de la información de la fase, con variaciones en la posición del objeto con respecto a la fuente de emisión y reflexión de la onda electromagnética. El desplazamiento medido $d$ del objeto bajo investigación es derivado de la información de variación de fase $\Delta \varphi$ medido por los sensores del radar en varios momentos de adquisición de información (Talich, 2013). El desplazamiento $d$ y la variación de fase $\Delta \varphi$ medidos están vinculados por:

$$
d=-\frac{\lambda}{4 \pi} \Delta \varphi
$$

El principio del funcionamiento de la interferometría aparece ilustrado en la Figura 4.

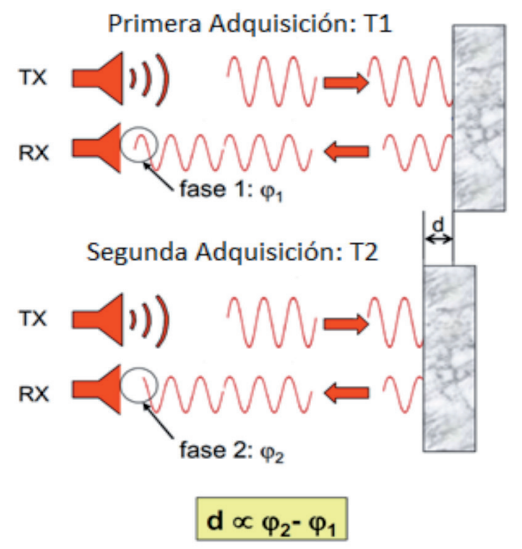

Figura 4: Principio de funcionamiento de la técnica interferométrica (Talich, 2013)

\section{Aplicación de pruebas de carga estática}

Para la aplicación de la prueba de carga estática se tomó como elemento a controlar el vano que salva el segundo tramo del puente correspondiente con la senda de circulación de la autopista en el sentido Habana Pinar (Figura 5). Para controlar el mismo se colocó el equipo de medición de forma tal que se adquirieran los desplazamientos experimentados en el centro de la luz y los apoyos del tramo, tal como se sugiere en la norma cubana NC 53-07 (2014) y las normativas de pruebas de carga de referencia internacional DIN 4150-3 (1999).

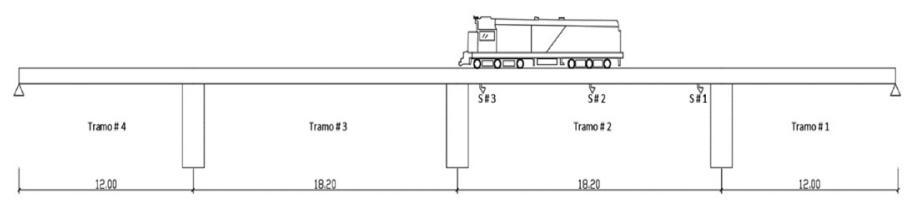

Figura 5: Localización del tramo medido en el puente (dimensiones en $\mathrm{m}$ )

Para aplicar la carga de prueba se colocó la locomotora de manera tal que el truck delantero se ubicara sobre el centro de la luz del tramo a controlar (Figura 6). Siendo esta la posición de la locomotora que provoca los mayores esfuerzos de flexión, según lo calculado mediante las líneas de influencia en diferentes secciones de las vigas del puente.

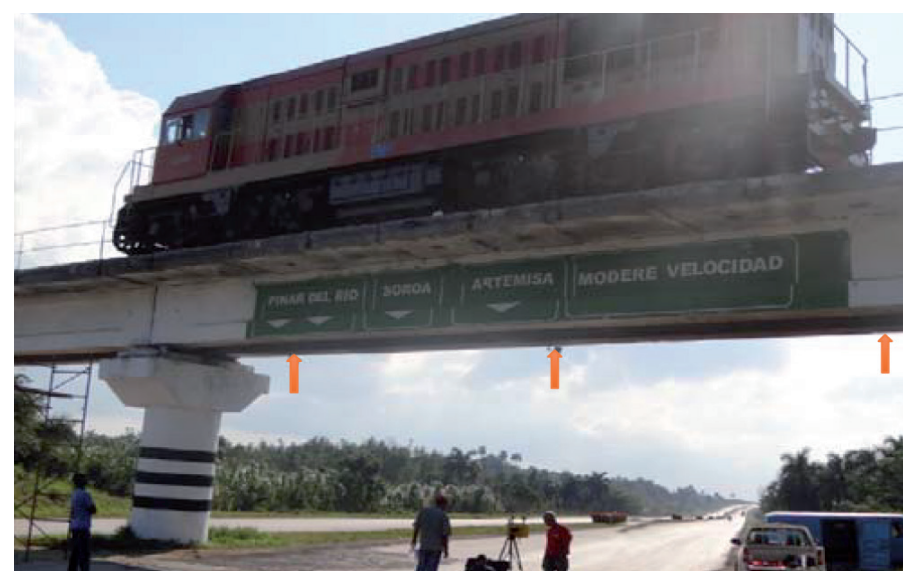

Figura 6: Aplicación de prueba de carga estática ( $\uparrow$ puntos controlados)

El proceso de monitoreo comenzó con el puente descargado, posteriormente se aplicó la carga durante un tiempo de 10 min y finalmente la carga fue retirada del tramo de la estructura comprobado. El experimento fue repetido dos 
veces con el objetivo de replicar el ensayo y obtener el promedio de las mediciones obtenidas. Durante la prueba se midieron de manera ininterrumpida los desplazamientos experimentados por el puente durante la aplicación de la carga y posteriormente durante el procesamiento de la información obtenida se calcularon los valores de la recuperación de las deformaciones experimentadas con el objetivo de determinar el intervalo de trabajo del puente.

El valor máximo de desplazamiento vertical obtenido en el centro de la luz fue de $3.35 \mathrm{~mm}$, tal como puede observarse en la Figura 7, siendo este valor 11 veces menor que 37.5 $\mathrm{mm}$, valor límite establecido para el desplazamiento en este puente en la norma cubana NC-207 (2003), donde se plantea que este valor se determina dividiendo la luz de trabajo del puente entre 450 .

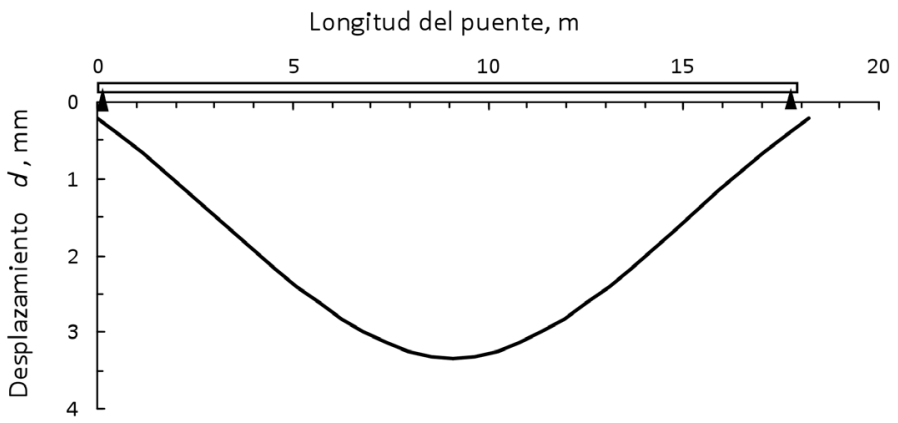

Figura 7: Gráfico de desplazamientos a lo largo del puente

A partir de los valores de desplazamiento experimentados durante el proceso de retirada de la carga se calcularon los valores de la recuperación de los desplazamientos donde se observa que el valor promedio de la recuperación es de $80.95 \%$, lo que indica que el puente se encuentra trabajando en la etapa elástica del material a partir de los criterios establecidos en la norma cubana NC 53-07 (2014).

\section{Aplicación de pruebas de carga dinámica}

Para la aplicación de la prueba de carga dinámica se mantuvieron los mismos puntos controlados variando la forma de aplicación de la carga, siendo la misma el paso de la locomotora varias veces a velocidades de 40,60 y $80 \mathrm{~km} / \mathrm{h}$. En cada medición se tomaron lecturas $5 \mathrm{~min}$ antes del paso de la locomotora y 5 min después del paso de la locomotora con el objetivo de describir de manera completa el proceso de vibración del puente ante el paso de la fuente excitadora.

\section{Determinación de las amplitudes vibratorias}

En las mediciones se obtuvieron las amplitudes de respuesta en términos de velocidad expresada en mm/s (Figura 8).

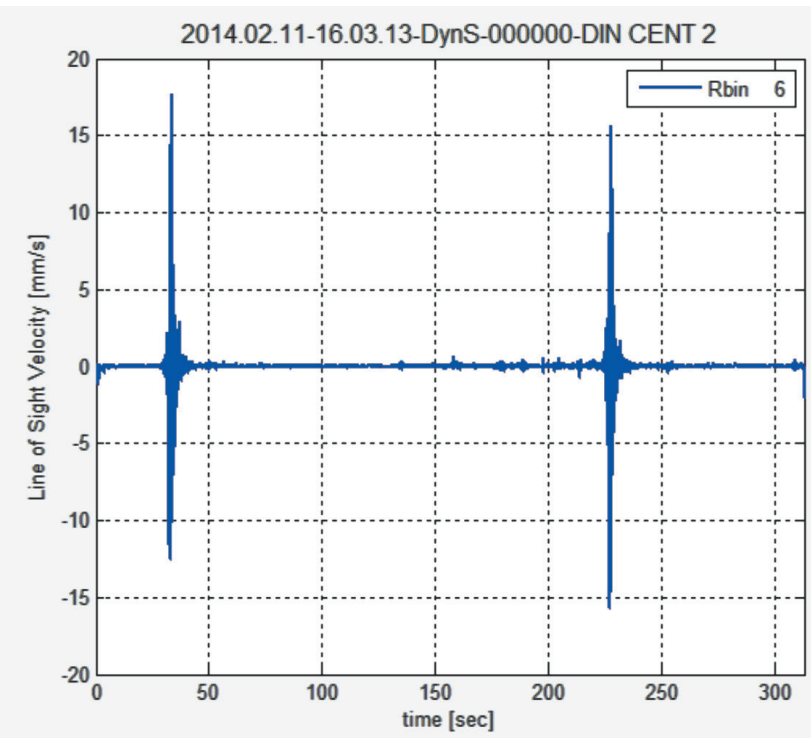

Figura 8: Espectros de velocidad obtenidos para los pasos de máquina a 60 (primer máximo) y a $80 \mathrm{~km} / \mathrm{h}$ (segundo máximo)

Como se observa el valor máximo de la velocidad de vibración en la dirección vertical obtenido fue de $16 \mathrm{~mm} / \mathrm{s}$, valor que indica un adecuado comportamiento dinámico de la estructura, según lo planteado en el apartado 5.20 de la norma DIN 4150-3 (1999), donde se establece que para las estructuras como el puente estudiado, el valor de velocidad de vibración en la dirección vertical debe ser inferior a 20 $\mathrm{mm} / \mathrm{s}$. También a partir del procesamiento de la señal de velocidad obtenida se calculó la razón de amortiguamiento (damping ratio) según la fórmula (2) como una función de la diferencia de amplitud entre dos crestas sucesivas de una medición (Chopra, 1980).

$\xi=\frac{\ln \frac{u_{i}}{u_{i+j}}}{2 \pi j}$

El valor de relación de amortiguamiento obtenido fue de 0.047 lo que significa que la estructura requiere de 2.5 ciclos de vibración para reducir el 50\% de la amplitud de las oscilaciones (Figura 9). 


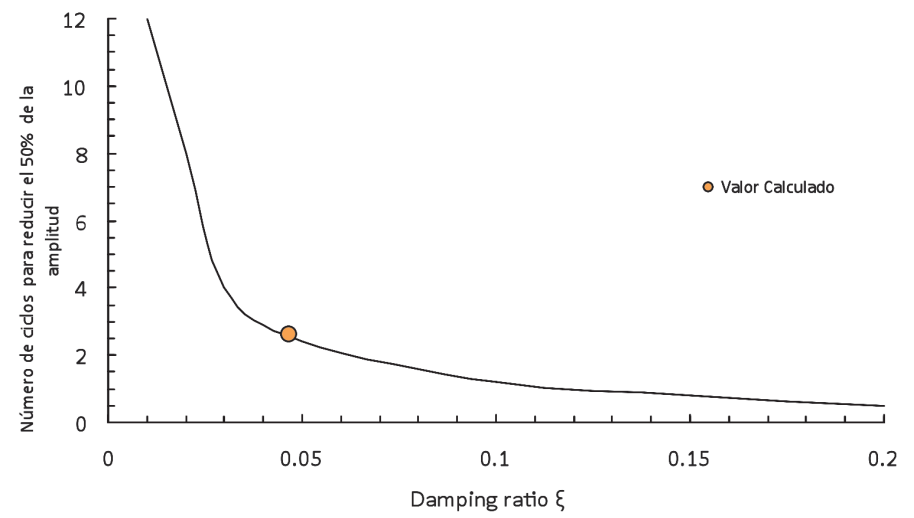

Figura 9: Número de ciclos requeridos para reducir el 50\% de la amplitud de la oscilación en función del amortiguamiento (Chopra, 1980)

\section{Identificación de las frecuencias de oscilación}

Mediante el procesamiento de las señales obtenidas de velocidad de vibración en el dominio del tiempo por medio de la aplicación de la Transformada Rápida de Fourier FFT a cada una de las señales, se obtienen estas expresadas en el dominio de la frecuencia (Figura 10). Esto permite la identificación de cada una de las frecuencias y periodos fundamentales de oscilación del puente.

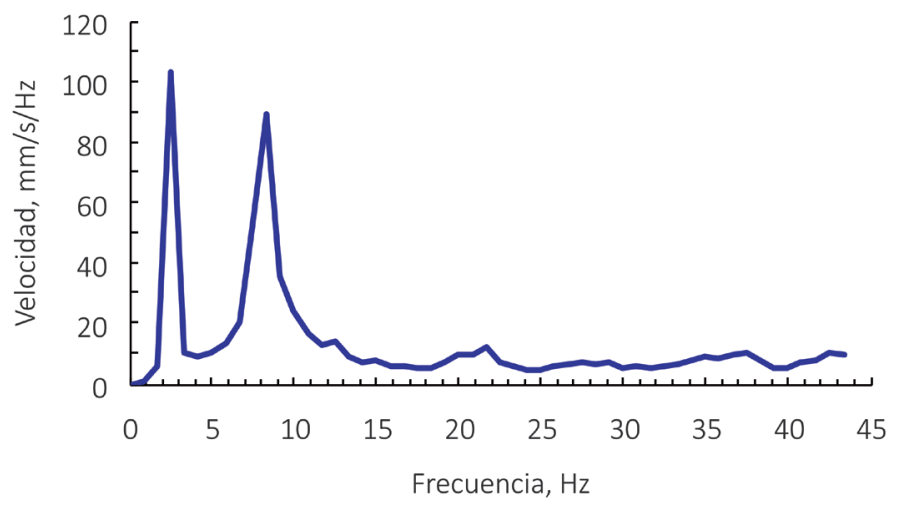

Figura 10: Espectro de potencias en velocidades

Es válido destacar que debido a las condiciones de las mediciones realizadas no fue posible identificar todos los modos de flexión del puente, solo algunos, es decir, sus frecuencias correspondientes. Tampoco fue posible distinguir los modos torsionales ni identificar modos de oscilación de flexión de varias luces acopladas mediante las mediciones, puesto que solo fue instrumentada una sola parte del puente y en una misma luz a lo largo de una alineación o viga. Este hecho no invalida los resultados del proceso investigativo. La existencia de modos de flexión producto del acoplamiento entre las luces es muy poco probable en este caso debido a que la continuidad entre los vanos es mínima, dada únicamente por la presencia de la capa de balasto y los propios rieles de la vía.

La identificación de los modos torsionales solamente influiría en el caso de que existieran dos carriles sobre el puente y una forma modal con una frecuencia menor que la identificada como fundamental que pudiera provocar la existencia de un efecto de resonancia ante el paso de los trenes a una menor velocidad por uno solo de los carriles (Cremona et al., 2009).

En el procesamiento de la señal obtenida se identificó como frecuencia fundamental de oscilación del puente $3.14 \mathrm{~Hz}$ y su período correspondiente posee un valor de $0.32 \mathrm{~s}$. También fueron identificados otras frecuencias y períodos propios de vibración los cuales se muestran en la Tabla 1.

Tabla 1: Parámetros de los modos propios de oscilación del puente

\begin{tabular}{|c|c|c|l|}
\hline No & Frecuencia, $\mathrm{Hz}$ & Período, $\mathrm{s}$ & Observaciones \\
\hline 1 & 3.14 & 0.32 & Fundamental, acoplamiento \\
\hline 2 & 8.33 & 0.12 & $\begin{array}{l}\text { Flexional, acoplamiento } \\
\text { ligero }\end{array}$ \\
\hline 3 & 21.66 & 0.05 & Flexional con acoplamiento \\
\hline 4 & 37.50 & 0.03 & Flexional con acoplamiento \\
\hline
\end{tabular}

\section{Modelización de la estructura}

Para la creación del modelo de la estructura fueron tomados los datos de proyecto para la introducción de las características geométricas de cada uno de los elementos, así como para definir las condiciones de frontera. Mientras que para definir las propiedades de los materiales componentes de cada elemento fueron tenidos en cuenta los resultados de los ensayos de caracterización practicados y descritos con anterioridad. Teniendo en cuenta que el software utilizado, MIDAS CIVIL 2013, realiza los cálculos estructurales por el MEF (MIDAS Family Programs, 2013), se realiza un proceso de refinamiento u optimización del mallado, que consiste en dividir los elementos en elementos cada vez más pequeños hasta obtener la densidad de mallado más adecuada. Mientras más fina sea la malla el resultado obtenido tendrá más precisión, pero tiene el inconveniente de tener un mayor costo computacional, por lo que es 
necesario encontrar la densidad de malla óptima a partir de la cual el modelo no ofrece variaciones significativas de una variable respuesta. Para realizar este análisis se toma como variable respuesta el corrimiento o desplazamiento en un punto bajo la acción de las cargas de peso propio de la estructura obtenidas para diferentes densidades de mallado (tamaños de cada uno de los elementos finitos de la malla), identificándose como densidad óptima a la densidad a partir de la cual los desplazamientos comienzan a adoptar un comportamiento asintótico (Figura 11) y la diferencia entre el desplazamiento obtenido por esa densidad de malla y la siguiente es menor del 5\%. Definiéndose finalmente como densidad de mallado óptima la malla de $25 \mathrm{~cm}$ x 25 $\mathrm{cm}$.

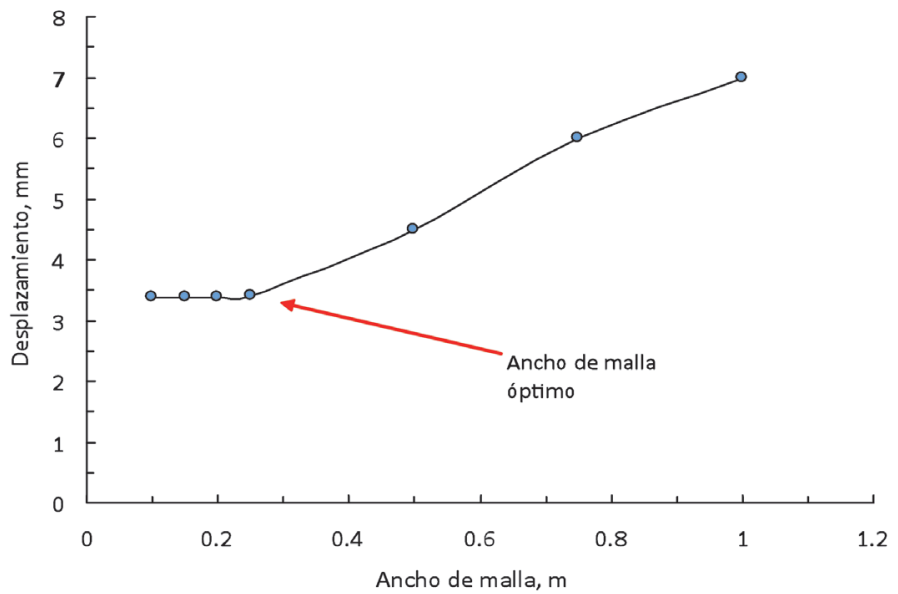

Figura 11: Selección del ancho de malla óptimo para la creación del modelo.

Para la modelización de la carga tributada desde el punto de vista dinámico del tren al puente fue analizada la interacción vehículo-estructura bajo el concepto o principio de la aplicación de fuerzas de contacto. Este método tiene en cuenta para la formulación del valor de la carga a aplicar, las características de amortiguamiento y de desplazamientos propios del vehículo que vaya a ser evaluado en el análisis (Yang et al., 2004). También se plantea la aplicación teniendo en cuenta su efecto en el tiempo en cada uno de los puntos de aplicación de la misma, por lo que, los valores de la carga han de ser introducidas en los cálculos en forma de funciones del tiempo (Figura12). Los resultados de diferentes modelos de interacción vehículo- estructura que han utilizado este método han sido validados mediante los resultados de numerosas pruebas de carga, donde se ha observado un alto grado de correspondencia entre los resultados de los modelos de cálculo y las pruebas de carga (Yang et al., 2004).

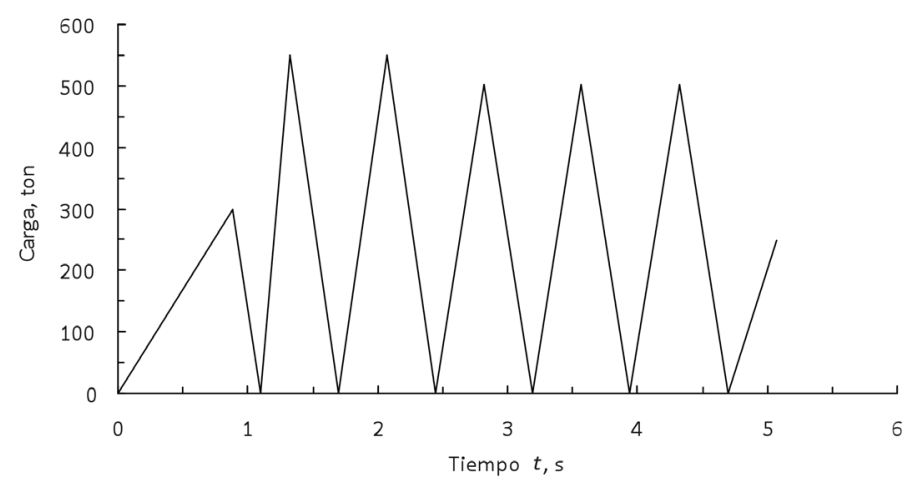

Figura 12: Modelación del paso del tren estudiado, ejemplo a $80 \mathrm{~km} / \mathrm{h}$

\section{Validación del modelo}

La respuesta del puente ante la acción de diferentes cargas no evaluadas durante el proceso de pruebas, serán calculadas con el modelo computacional creado. Por lo que se hace necesaria la realización de un proceso de ajuste de las variables del modelo, para ajustar el mismo a la realidad de respuesta de la estructura del puente. Para ello son comparados los valores de respuesta del modelo ante la acción de las cargas aplicadas durante las pruebas con los resultados obtenidos en las pruebas estáticas, donde se obtuvo una concordancia del orden del $96.2 \%$ entre los resultados brindados por el modelo y los resultados de las pruebas estáticas (Figura 13).

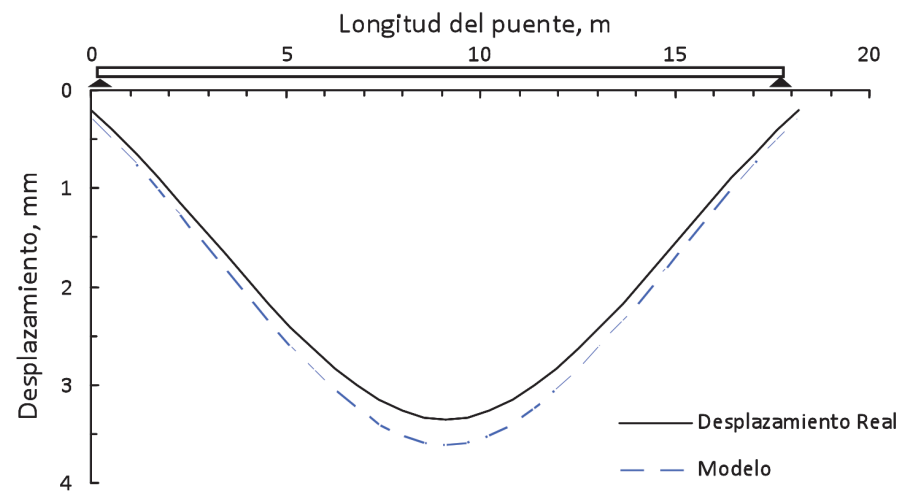

Figura 13: Gráficos de los valores de desplazamiento obtenidos

También desde el punto de vista del análisis dinámico se realizó la comprobación de ajuste del modelo a los valores 
obtenidos en las mediciones. Para ello, los resultados bridados por los modelos dinámicos realizados fueron comparados con los resultados medidos de manera directa durante el proceso de realización de pruebas de carga, mostrando los mismos un alto nivel de correspondencia con los valores reales medidos, puesto que en el modelo se obtienen oscilaciones que a pesar de estar desfasadas en el tiempo con respecto a las oscilaciones medidas, poseen amplitudes similares con un nivel de correspondencia del 96\% entre ellas (Figura 14).

(a)

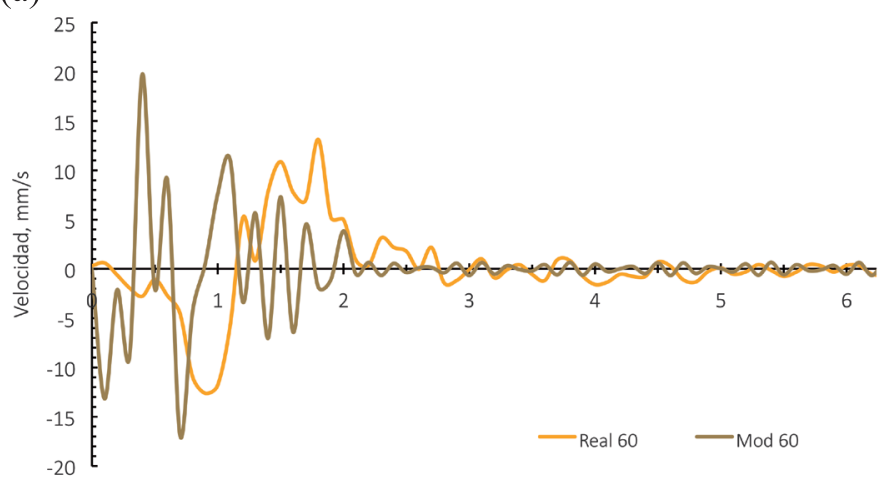

(b)

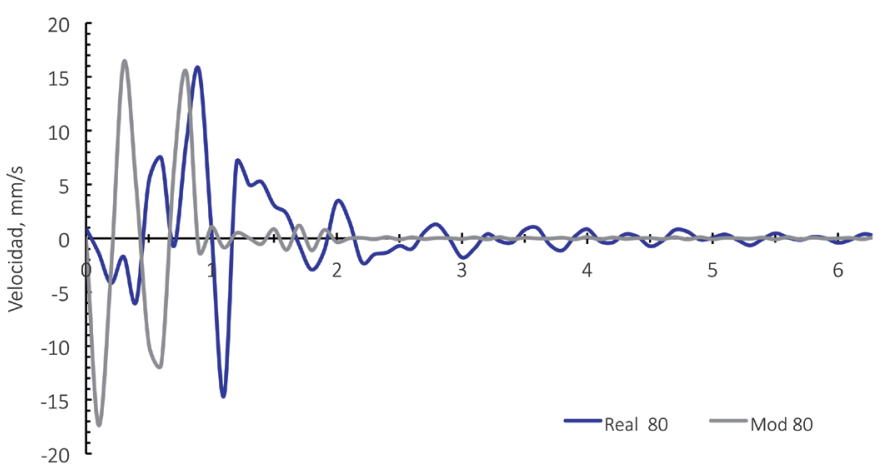

Figura 14: Gráficos de las oscilaciones experimentadas por el puente para a) $60 \mathrm{y} \mathrm{b)} 80 \mathrm{~km} / \mathrm{h}$

\section{Análisis de los resultados de la modelación}

\section{Identificación modal}

Para la obtención de las formas modales de la estructura se realizó un análisis mediante la aplicación del método Lanczos en el cual fueron determinadas las formas de oscilación que aparecen en la Figura 15.

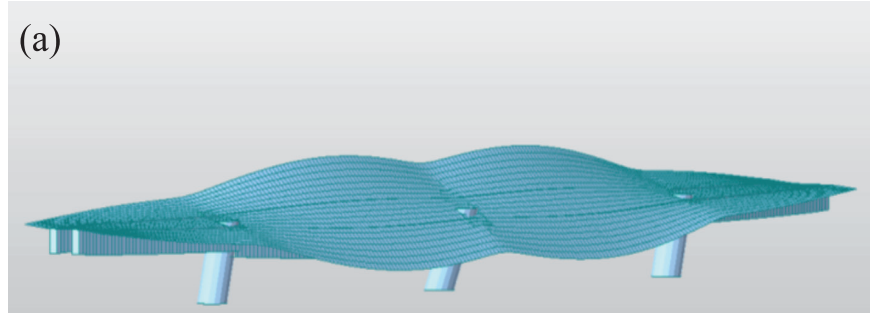

(b)

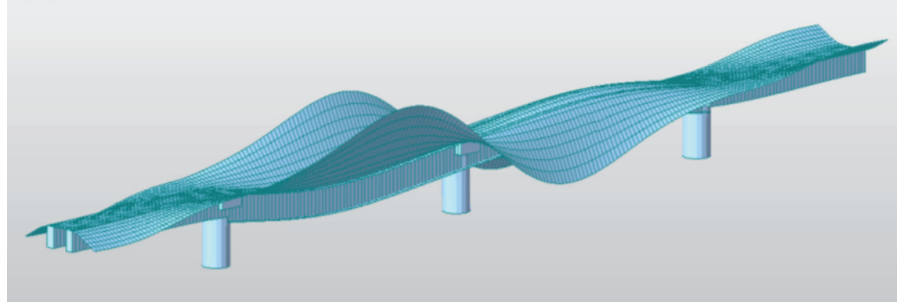

(c)

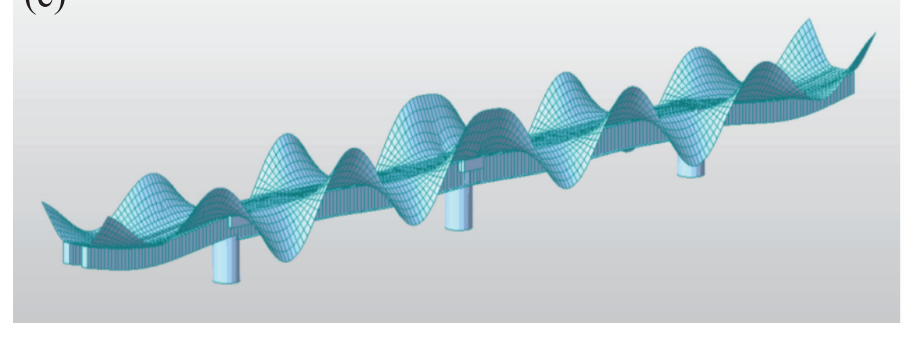

(d)

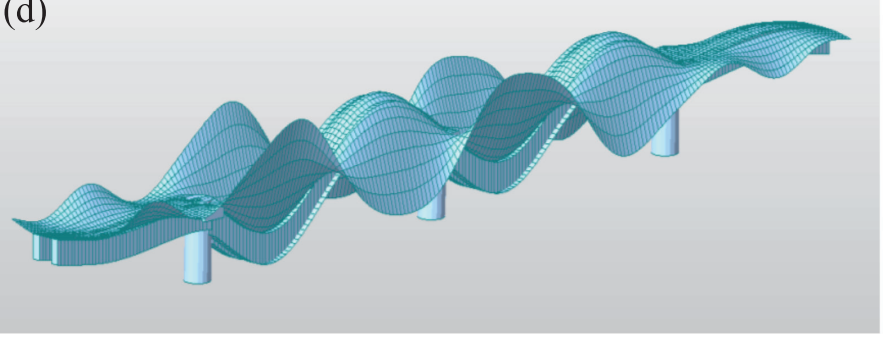

Figura 15: Formas modales identificadas, a) modo 1: frecuencia de $3.14 \mathrm{~Hz}$ y período de $0.32 \mathrm{~s}, \mathrm{~b}$ ) modo $2: 8.28 \mathrm{~Hz}$ y $0.12 \mathrm{~s}$, c) modo $3,21.54 \mathrm{~Hz}$ y $0.05 \mathrm{~s}$ y d) modo $4: 37.59 \mathrm{~Hz}$ y $0.03 \mathrm{~s}$

Tabla 2: Comparación de características modales calculadas y medidas

\begin{tabular}{|c|c|c|c|c|}
\hline \multirow{2}{*}{ Modos } & \multicolumn{2}{|c|}{ Valores medidos } & \multicolumn{2}{c|}{ Valores calculados } \\
\cline { 2 - 5 } & $\begin{array}{c}\text { Frecuencia, } \\
\mathrm{Hz}\end{array}$ & Periodo, $\mathrm{s}$ & $\begin{array}{c}\text { Frecuencia, } \\
\mathrm{Hz}\end{array}$ & Periodo, $\mathrm{s}$ \\
\hline 1 & 3.14 & 0.32 & 3.142 & 0.318 \\
\hline 2 & 8.33 & 0.12 & 8.280 & 0.120 \\
\hline 3 & 21.66 & 0.05 & 21.54 & 0.120 \\
\hline 4 & 37.50 & 0.03 & 37.59 & 0.027 \\
\hline
\end{tabular}


Es válido destacar que entre las frecuencias propias identificadas en las mediciones y las identificadas mediante los cálculos de identificación modal existe un alto nivel de correspondencia lo que denota la efectividad del método de identificación modal utilizado (Tabla 2).

\section{Análisis estático}

La respuesta del puente a las diferentes cargas (peso propio, viento, carga viva) fue calculada con el modelo calibrado. Los resultados de estos cálculos fueron usados para verificar la capacidad de carga y de servicio en los diferentes elementos. La condición de carga muerta fue calculada tomando en cuenta las fases de construcción, la instalación de los cables de pre-esfuerzo y el peso del balasto y la vía. Para la realización del proceso de revisión estructural se calcularon las capacidades resistentes de cada uno de los elementos a partir de los datos obtenidos durante el proceso investigativo, utilizando como principios de cálculo de elementos de hormigón armado los procedimientos propuestos por el ACI 318 (2008). Calculado el valor del momento nominal resistente (4500 $\mathrm{kNm}$ ) se comprobó el cumplimiento de la condición $\phi M_{\mathrm{n}} \geq$ $M_{\mathrm{u}}$, la cual vela porque el valor minorado de la resistencia nominal a flexión sea mayor que el valor del momento último mayorado actuante sobre la pieza. En el caso del puente estudiado los valores del momento flector actuante $(3800 \mathrm{kNm})$ no superan a los valores del momento flector nominal resistente de la sección, mostrando la sección estudiada un coeficiente de aprovechamiento de la sección (ratio $\mathrm{K}$ ) $\mathrm{K}=0.85$ lo que significa que la sección principal del puente trabaja al $85 \%$ de su capacidad. De igual modo se comprobaron los esfuerzos cortantes y los estados límites de fisuración y deformación, determinándose que la sección resiste satisfactoriamente los esfuerzos provocados por las cargas que sobre el puente actúan.

\section{Análisis dinámico}

La caracterización de la respuesta dinámica de la estructura permite la evaluación del efecto del paso de los trenes por el puente a diferentes velocidades de circulación. Para el análisis se modeló primeramente el paso de la locomotora utilizada en las pruebas con el fin de comparar los resultados del modelo con los valores reales medidos, para de esta forma evaluar los niveles de correspondencia existentes entre los resultados brindados por el modelo y los realmente obtenidos del comportamiento del puente. Una vez comprobada la correspondencia entre el modelo y la realidad se modeló el paso de los trenes completos, para lo cual se tomaron como dato los trenes tipo establecidos en IAPF (2001). También se evaluó el efecto de la generación de resonancia, este fenómeno ocurre cuando se sintoniza alguna de las frecuencias de excitación con alguna frecuencia natural de la estructura, en estos casos las amplitudes de las oscilaciones son notablemente amplificadas. Este fenómeno representa gran importancia en el caso del análisis de estructuras, puesto que al entrar en resonancia, los esfuerzos experimentados se multiplican al punto de poder generarse el colapso total o parcial de las edificaciones.

En el proceso de modelación fueron evaluados los pasos de los trenes a velocidades de 40, 60, 80 y $110 \mathrm{~km} / \mathrm{h}$ teniendo en cuenta el efecto vibratorio de cada una de estas velocidades a partir del cálculo de la frecuencia y período de excitación que provocan cada una de estas velocidades. Estos valores de frecuencia y período de excitación fueron calculados para la locomotora y para los coches evaluados a partir de las dimensiones y cargas características (Tabla 3).

Tabla 3: Frecuencias y períodos de excitación para cada velocidad evaluada

\begin{tabular}{|c|c|c|c|c|c|}
\hline \multicolumn{3}{|c|}{ Locomotora } & \multicolumn{3}{c|}{ Coches } \\
\hline $\begin{array}{c}\text { Velocidad } \\
\mathrm{km} / \mathrm{h}\end{array}$ & $\begin{array}{c}\text { Frecuencia, } \\
\mathrm{Hz}\end{array}$ & Periodo, $\mathrm{s}$ & $\begin{array}{c}\text { Velocidad } \\
\mathrm{km} / \mathrm{h}\end{array}$ & $\begin{array}{c}\text { Frecuencia, } \\
\mathrm{Hz}\end{array}$ & Periodo, $\mathrm{s}$ \\
\hline 40 & 1.13 & 0.88 & 40 & 0.67 & 1.50 \\
\hline 60 & 1.70 & 0.59 & 60 & 1.00 & 1.00 \\
\hline 80 & 2.26 & 0.44 & 80 & 1.33 & 0.75 \\
\hline 110 & 3.11 & 0.32 & 110 & 1.83 & 0.55 \\
\hline
\end{tabular}

También fue modelado el paso de la locomotora a $110 \mathrm{~km} / \mathrm{h}$ que es la velocidad que provoca un valor de frecuencia generada $(3.11 \mathrm{~Hz})$ similar a la frecuencia fundamental de vibración de la estructura, identificada mediante el proceso de mediciones dinámicas. La Figura 16 muestra los resultados del análisis de esta variante, donde se observa un aumento considerable de la amplitud de las oscilaciones mostrándose un crecimiento del orden de 5.5 veces el valor de la amplitud de las oscilaciones provocadas por el paso de la máquina a 40, 60 y $80 \mathrm{~km} / \mathrm{h}$, lo que demuestra la entrada en resonancia del puente ante la variante de carga evaluada (paso a $110 \mathrm{~km} / \mathrm{h}$ ).

Mediante la realización de diferentes corridas del modelo 
fueron evaluados los efectos del paso de la locomotora sola a 40, 60 y $80 \mathrm{~km} / \mathrm{h}$ donde se obtuvieron magnitudes de esfuerzos que son inferiores a los valores límites de resistencia ante los esfuerzos de la estructura (Figura 18).

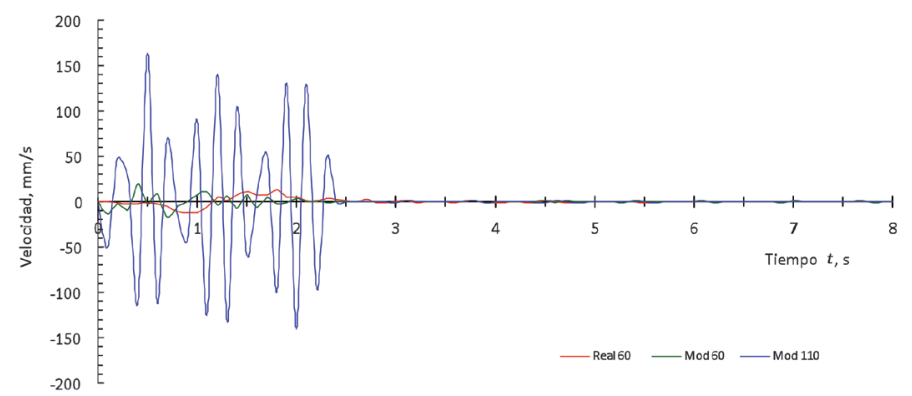

Figura 16: Oscilaciones experimentadas por el paso de la locomotora a 60,80 y $110 \mathrm{~km} / \mathrm{h}$

Para la evaluación del paso del tren entero se conformó un tren tipo, según los parámetros establecidos por IAPF (2001), compuesto por 10 coches de carga con capacidad para $1000 \mathrm{kN}$ de carga y la locomotora utilizada durante el estudio. Se realizaron corridas donde se evaluó el paso de este tren a velocidades de 70,80 y $110 \mathrm{~km} / \mathrm{h}$. Los resultados obtenidos son mostrados en la Figura 17, donde se muestra que los momentos provocados por el paso del tren a $110 \mathrm{~km} / \mathrm{h}$ sobrepasan el límite del colapso de la estructura, por su parte los momentos provocados por el paso del tren a $80 \mathrm{~km} / \mathrm{h}$ sobrepasan el límite de la seguridad estructural, mientras que se demuestra que el puente resiste satisfactoriamente el paso del tren a $70 \mathrm{~km} / \mathrm{h}$. Por estas razones se propone el establecimiento de la velocidad de $70 \mathrm{~km} / \mathrm{h}$ como límite máximo de velocidad de circulación de los trenes sobre el puente.

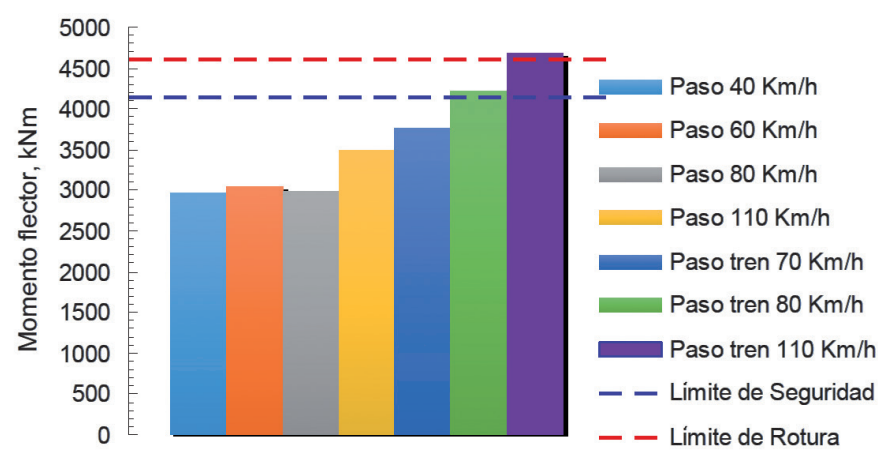

Figura 17: Comparación de momentos flectores generados por el paso de diferentes tipos de trenes

\section{Conclusiones}

A partir de la realización de los ensayos de carga estático se logró comprobar el correcto funcionamiento ya que el desplazamiento máximo medido de $37.5 \mathrm{~mm}$, es menor que el valor de la luz del vano dividido entre 450 . La recuperación final de los desplazamientos fue mayor del $75 \%$, los valores de espesores de fisuras medidos fueron menores que los valores normados como permisibles $(0.2$ $\mathrm{mm}$ ) y durante la realización de la prueba no se produjeron colapsos estructurales; tal como plantean los criterios de evaluación estructural establecidos en la NC 53-07 (2014).

A partir del valor máximo de amplitud de oscilación medido del puente de $16 \mathrm{~mm} / \mathrm{s}$, se puede afirmar que el comportamiento estructural es adecuado desde el punto de vista dinámico, ya que no se superan los $20 \mathrm{~mm} / \mathrm{s}$ que es el valor límite establecido por DIN 4150-3 (1999). Se identificó como período fundamental de oscilación del puente un período $\mathrm{T}=0.32 \mathrm{~s}$. Se identificó el paso de los trenes (locomotora + coches) a $110 \mathrm{~km} / \mathrm{h}$ como la velocidad que provoca la entrada en resonancia del puente. A partir de los resultados obtenidos se estableció la velocidad de 70 $\mathrm{km} / \mathrm{h}$ como límite máximo de velocidad de circulación de los trenes sobre el puente.

\section{Referencias}

Alvandi, A. and Cremona, C. (2002). Reliability of bridge integrity assessment by dynamic testing. European Conference on Structural Health Monitoring, Cachan, France

ACI 318 (2008). Building code requirements for structural concrete and commentary. American Concrete Institute. Farmington Hills, USA

Bathe, K.J. (2013). The subspace iteration method-revisited. Computers \& Structures 126, 177-183

Chopra, A. (2011). Dynamics of Structures. Fourth edition, Prentice Hall

Cremona, C., Yang, S. and Dieleman, L. (2009). Overview of feature extraction techniques. Proceedings of the Third International Operational Modal Analysis Conference, Portonovo, Italy. Vol. 2.

DIN 4150-3 (1999). Structural vibration. Effects of vibrations on structures. Deutsches Institut für Normung. Berlin: Beuth Verlag GMBH. 
Ewins, D.J.(2000). Modal testing: theory, practice and application. Research Studies Press LTD., Baldock, Hertfordshire, England 171: $415-437$

IAPF (2001). Instrucción de acciones a considerar en el proyecto de puentes de ferrocarril. Ministerio de Fomento, España

IDS-Ingenieria del Sistemi (2013). IBIS Surveyor v.01.00. User Manual. Pisa: IDS Ingegneria Dei Sistemi S.p.A.

Kiseliov, V.A. (1983). Mecánica de la construcción. Dinámica y estabilidad de las estructuras. Curso Especial. Moscú: Mir

Lanczos, C. (1950). An iteration method for the solution of the eigenvalue problem of linear differential and integral operators. Journal of Research of the National Bureau of Standards 45, 255-282

MIDAS Family Programs (2013). Getting Started Manual. Seul: MIDAS IT Co.

NC 207 (2003). Requisitos generales para el diseño y construcción de estructuras de hormigón. Norma Técnica. Comité Estatal de la Construcción, Oficina Nacional de Normalización, La Habana, Cuba
NC 53-07 (2014). Estructuras de hormigón-pruebas de carga directa. Norma Técnica. Comité Estatal de la Construcción, Oficina Nacional de Normalización, La Habana, Cuba

Red DURAR (1997). Manual de inspección, evaluación y diagnóstico de corrosión en estructuras de hormigón armado. Programa Iberoamericano de Ciencia y Tecnología para el Desarrollo, Subprograma XV Corrosión/Impacto Ambiental sobre Materiales, CYTED, Maracaibo

Talich, M. (2013). Possibilities of precise determining of deformation and vertical deflection of structures using ground radar interferometry. FIG Working Week. From the Wisdom of the Ages to the Challenges of the Modern World. Sofia, Bulgaria, 17-21 May 2015

Yang, Y.B., Yau, J.D. and Wu, Y.S. (2004). Vehicle-Bridge Interaction Dynamics. With Applications to High-Speed Railways. World Scientific Publishing. 